\title{
Category-structure effects in picture comparisons
}

\author{
ROBERTA L. KLATZKY and ANN STOY RYAN \\ University of California, Santa Barbara, California 93106
}

\begin{abstract}
Subjects indicated for a series of trials whether or not two pictures of common objects were from the same category. Reaction time (RT) was recorded. The pool of stimuli used in the series of trials consisted of two pictures representing each of several categories. Half were S categories, members of which were similar; the other half were D categories, which had dissimilar members (this variable is termed category structure). In Experiments 1 and 3, the similarity manipulation defining category structure was based on the concept of basic level categories (Rosch, Mervis, Gray, Johnson, \& Boyes-Braem, 1976). In Experiment 2, category labels were homonyms, and the two members of $S$ or $\mathrm{D}$ categories represented one or two senses of the labels, respectively. The principal finding was an effect of category structure (faster RT on trials using stimuli from $\mathrm{S}$ categories rather than D categories). These results are interpreted in terms of a model that postulates the use of semantic codes in the task, even on trials where the two stimuli are physically identical.
\end{abstract}

The representation of visual information in memory has been the topic of considerable research. Many of the recent advances in this area have been contributed by Posner (Posner, Boies, Eichelman, \& Taylor, 1969; Posner \& Mitchell, 1967), using a task which requires forms to be compared. In one variant of the task, the subject decides on each trial whether two visual stimuli (e.g., letters) have the same or different names and makes a positive or negative response, respectively. Two types of positive trial can occur: The stimuli can be physically identical (an identity match) or nonidentical but have the same name (a category match). A general finding is that reaction time (RT) for identity matches is less than that for category matches, as long as the stimuli occur within a few seconds of one another. Posner has interpreted this result as indicating that different codes are used for the two types of match-visual codes for the identity match and name codes for the category match. The research presented here extends those findings in a task which requires temporally separated pictures of common objects to be compared. A model is proposed for this task that presents a somewhat different view of the task than the usual visual/verbal dichotomy.

The proposed model draws on findings from other studies that use temporally separated stimuli in the task developed by Posner. When the two stimuli are separated in time, the comparison process logically must utilize a representation in memory of the first

This research was conducted with the aid of Grant MH25090 from the National Institute of Mental Health. The authors thank John Cotton and John Ernest for their suggestions about the model and Russ Revlin for his comments on the manuscript. stimulus. Under these conditions, identity matches still have been found to be faster than category matches, suggesting that the former utilize a visual memory code of the first stimulus presented. However, as the interstimulus interval (ISI) increases, identity-match RT gradually increases until it reaches category-match RT. This finding suggests that the visual memory code is a short-term representation, from which information is lost over time.

Other findings with this paradigm suggest that the short-term visual code of the first stimulus, used for identity matches, is more abstract than a sensory code. For one thing, the difference between category and identity matches is unaffected by the introduction of a masking field during the ISI, which would eliminate any sensory trace of the first stimulus (Posner, 1969). In addition, Klatzky and Stoy (1974) found that the time to match two identical pictures of objects was no faster than the time to match two pictures that were mirror images of one another. If the short-term code of the first picture was a veridical image, the configurational differences between mirror-reversed stimuli would be expected to lead to slower RTs.

One additional postulated characteristic of this short-term visual memory is that there can be generated, from information in long-term memory, a representation that resembles the memory code retained shortly after presentation of a visual stimulus. This is indicated by an experiment in which subjects were given either an auditory (spoken name) or visual presentation of the first letter, followed after an interval by a visual letter (Posner et al., 1969). With a 1-sec ISI, two identical visual letters were matched no faster than an initial auditory letter and 
a second visual one. This finding and those of related studies (e.g., Cooper \& Shepard, 1973; Tversky, 1969) suggest that subjects can use a label to access long-term memory and generate a visual representation of the corresponding stimulus.

In the model of the stimulus-comparison task presented here, the concept of generation plays a central role. Specifically, the model assumes that category matches, and some identity matches, utilize stimulus representations generated from long-term memory. A similar view of the task has been adopted by Kroll and his colleagues (Kroll, 1975; Parks \& Kroll, 1975). Instead of assuming that physical and nominal codes are used for identity and category matches, respectively, they have proposed that, given the first stimulus of a trial, the subject generates a visual code which then underlies category matches. In support of this view, Parks and Kroll (1975) manipulated task variables that might make the generation of alternative stimuli in the category more difficult. For example, they increased the number of the stimuli in each category; this resulted in a longer-lasting advantage of identity over category matches, supporting the generation hypothesis. And this occurred even when the subject had to rehearse the name of the firststimulus category throughout the ISI, ensuring the availability of a nominal code.

Other support for the hypothesis that category matches are based on abstract visual codes rather than names was offered by Klatzky and Stoy (1974), who used pictures of common objects as stimuli in the comparison task. In their studies, in addition to manipulating trial type and ISI, they manipulated a variable to be called "category structure." This refers to the fact that each subject in the experiment had experience with a pool of two-picture categories (e.g., two zebras, two lions, etc.). The two pictures composing the category were physically similar in the case of half of these categories; for the remaining categories, the two pictures were dissimilar. (These will be called S and D categories, respectively.) The difference between types of categories forming the stimulus pool meant that the RT data could be broken down according to the category membership of the stimuli that occurred. For example, an identity match could involve the repeated presentation of a member of an S category, or of a D category; similarly, a category match could utilize the two members of an S category, or of a D category. Klatzky and Stoy found that category structure affected RT for both identity and category matches, with greater RT on trials utilizing stimuli from a $\mathrm{D}$ category than those using S-category stimuli. In control conditions, however, in which subjects knew they should expect just one type of positive trial-identity or categorythere was no effect of the category-structure variable.
The important implication of the categorystructure effect is that the subject's processing of the stimuli on a given trial reflects his knowledge of the category from which they come. This is particularly indicated by the fact that the type of category from which the pictures come affects RT even on identity-match trials, where the two stimuli are always identical regardless of the nature of their category. A second important implication is that category matches in this experiment were based not on nominal encodings, but on some other abstract code containing information about both members of the category. Since category names should match equally well for S and D categories, a nominal match would not produce a category-structure effect.

The purpose of the first two experiments in the present paper is to extend the category-structure effects of Klatzky and Stoy (1974) to two new cases. In their original study, the category-structure variable manipulated the similarity of category members with respect to shape. However, the manipulation of shape may also have produced a manipulation of semantic properties. For example, the category zebra in their study was a D category and consisted of pictures of a zebra's head and a whole zebra. A semantic property such as "can run" may apply to the latter but not the former. This suggests that semantic variations may have played a role in the initial category-structure effects. The category-structure manipulations of the present study were explicitly based on semantic rather than physical properties, in order to determine whether such manipulations could also produce the effects.

In the first study, the similarity relation used to design stimulus categories was derived from distinctions made by Rosch and her associates (Rosch, Mervis, Gray, Johnson, \& Boyes-Braem, 1976) among different levels within categorical hierarchies. For several such hierarchies, for example, sports$\mathrm{car} / \mathrm{car} / \mathrm{vehicle}$, Rosch et al. have found there is a "basic" level (in the above example, car) at which category members have many attributes in common. At the superordinate level (vehicle), above the basic level, there are many fewer common attributes, and at subbasic levels (sports car), there are few more common attributes than at the basic level. Rosch et al. have found that basic-level categories have a special status in a variety of psychological tasks, such as picture naming, signal detection, and sorting by categories. In Experiment 1, each of the $S$ categories comprised two pictures from the same basic level and each of the D categories comprised two pictures from different basic levels but the same superordinate level.

In Experiment 2, the category-structure manipulation was based on homonyms: Members of S categories represented the same sense of their category label, 
whereas members of D categories represented different senses. This manipulation provides yet another extension of the original category-structure studies. Experiment 2 has an additional advantage as well: In Experiment 1, members of D categories may have differed with respect to the name subjects would implicitly give them (e.g., the members of a D category labeled "vehicle" might be pictures of a car and a truck); the S-category members would not differ nominally (e.g., the S-category "vehicle" might include two car pictures). This might occur even though the categories were overtly named by the experimenter and subject at the start of each session with the superordinate-level name ("vehicle"). In Experiment 2, however, the use of homonyms was intended to reduce the possibility that subjects would give different names to members of $\mathrm{D}$ categories.

\section{EXPERIMENTS 1 AND 2}

\section{Method}

Subjects. The subjects in each experiment were eight normal adults affiliated with the University of California, Santa Barbara. Different subjects were used in each experiment.

Stimuli. The stimulus materials were pictures (black-and-white drawings) representing familiar objects. Each picture was within approximately $5 \mathrm{~cm}$ in diameter. Throughout the study, each subject had experience with a pool of such stimuli, consisting of two pictures representing each of several categories (e.g., two vehicles; the categories are described below). For half the categories, the two pictures were similar with respect to a given criterion; this type of category will be termed an $\mathrm{S}$ category. The two pictures composing each of the remaining categories were dissimilar; a category of this type will be termed a D category.

The stimuli of Experiment 1 represented categories drawn from the nonbiological taxonomies of Rosch et al. (1976). The superordinate (and basic) level categories were: vehicle (car, truck), fruit (apple, grapes), clothing (shirt, socks), musical instrument (drum, piano), furniture (chair, lamp), and tool (hammer, screwdriver). Each subject was assigned a stimulus pool comprising two pictures from each of the six superordinate categories. Three of the categories each comprised two pictures from different subordinate-level categories but the same basic level (e.g., for a given subject, the category vehicle might include two cars-a sports car and a four-door sedan). These were the $S$ categories. The remaining categories each comprised two pictures from different basiclevel categories but the same superordinate level (e.g., the category musical instrument might include pictures of a grand piano and a kettle drum). These were the $\mathrm{D}$ categories. Thus the two pictures composing an $\mathrm{S}$ category were similar in that they belonged to the same basic-level category; the two pictures representing a D category were not similar in this respect. All six categories were named for the subject with the superordinate-level name (e.g., vehicle).

In Experiment 2, 10 categories were used. These categories were chosen because each of their names had two senses, represented identically in orthography and pronunciation but differing in meaning. The criterion for selection of these names was that each had two distinct dictionary definitions (according to Webster's Seventh Collegiate Dictionary; this definition of homonymy is based on Lyons', 1968). The 10 categories were: eye, glasses, hands, horn, keys, nail, nut, pipe, train, and trunk. To each subject, two pictures representing each of these 10 categories were assigned. For five of the categories, the two pictures represented the same sense of the category name (e.g., two pictures of pipes for smoking); these were the $\mathrm{S}$ categories. For the remaining five categories, the two pictures represented different senses of the category name (e.g., the keys to a door and pianc keys). These formed the $D$ categories.

In both experiments, although each subject had just two pictures from a given category in his stimulus pool, these two pictures were actually drawn from a larger set containing four pictures for each category. Each of the four pictures was similar to one other picture representing its category and dissimilar to the remaining two (e.g., in Experiment 2, there were two pictures of smoking pipes and two of plumbing pipes). From this larger pool of stimuli, pairs of pictures were assigned to individual subjects' stimulus pools within the constraint that each picture serve equally often as a member of an S category and a member of a D category, across subjects. As a result, when data were pooled over subjects, effects of variations in irrelevant properties in the individual pictures were counterbalanced.

Apparatus. The apparatus was a three-field Iconix tachistoscope. The subject sat at a table and looked into the exposure box, pressing a start bar with his thumbs to initiate each trial. The viewing distance was $90 \mathrm{~cm}$. The subject's index fingers were on two response switches, the preferred one of which was designated as the positive switch and the other the negative switch.

Procedure. Each subject participated in one practice session, followed by several experimental sessions (four in Experiment 1; seven in Experiment 2) of 120 trials each, each lasting about $1 \mathrm{~h}$. Each subject performed under three different types of instruction: With pure identity instructions, the subject was informed that only identity matches and negative trials would occur; there would be no category-match trials. With pure category instructions, he knew only category matches and negative trials could occur. With mixed instructions, the subject knew that category matches, identity matches, and negative trials could all occur. In Experiment 1 , the subject took part in two sessions with mixed instructions and two with pure instructions. In the latter case, half the session used pure identity and the other half pure category instructions. In Experiment 2, the subject took part in three sessions with mixed instructions, two with pure identity, and two with pure category instructions. The order of the experimental sessions was counterbalanced across subjects, with the constraint that no subject experience two consecutive sessions with the same instructions.

Within each session, trials using several ISIs occurred. The ISIs used in Experiment 1 were .25 and $3.0 \mathrm{sec}$; those used in Experiment 2 were $.25,1.75$, and $4.0 \mathrm{sec}$. The ISI was held constant for blocks of 10 trials and changed after each block, so that no ISI was repeated until the remaining ISIs had been used. The subject was informed before each block of trials as to which ISI would be used in that block. A few practice trials preceded each experimental session, and they used the same ISI as the first block of trials.

Within each session, for each ISI, positive and negative trials occurred in a 60:40 ratio in Experiment 1 and in a 50:50 ratio in Experiment 2. Each type of positive trial occurred approximately equally often. Stimuli from each type of category (S and $D$ ) occurred approximately equally often within each trial type. Within these constraints, the stimuli were randomly selected for each trial.

The practice session included a 10-trial block for each ISI, under each type of instruction. At the beginning of this session, the subject was shown pictures like those to be used in his stimulus pool and told their names. Thus the practice session gave the subject experience with the structure of all his stimulus categories as well as the nature of the RT task.

At the start of each session, the subject viewed arci named pictures like those in his stimulus pool; the series of trials thien began. The procedure for each trial was as follows: The subject pressed the start bar, causing a fixation field (four black dots forming the vertices of a $4.4-\mathrm{cm}$ square) to appear, centered in the viewing area, for $1.0 \mathrm{sec}$. The square vanished, and the first picture appeared for $.5 \mathrm{sec}$, centered in the area previously defined 
by the fixation square. The first picture then vanished and the fixation square reappeared for the ISI. The second stimulus then appeared, in the same position as the first. The subject pressed the positive or negative switch, according to whether or not the two pictures were from the same category. The experimenter informed the subject if he had made an error. Reaction time, defined as the interval between the onset of the second picture and the subject's response, was recorded.

\section{Results}

The principal data are mean RTs in milliseconds. The data analysis includes correct responses only; error rates were low, with a range of $.4 \%-3.2 \%$ and a mean of $1.8 \%$ in Experiment 1 and a range of $1.4 \%-4.2 \%$ and a mean of $2.9 \%$ in Experiment 2 . The errors were not subjected to detailed analysis, due to the small amount of data. However, it can be noted that error rates were generally positively correlated with RT, a result which contraindicates a speed/accuracy tradeoff. Data from practice sessions and from practice trials preceding each experimental session were not included in the analysis.

Figure 1 presents mean RT as a function of ISI under mixed instructions in the two experiments. Table 1 presents the corresponding data for pure instructions. These data are broken down by the category-structure variable ( $\mathrm{S}$ vs. $\mathrm{D}$; for negative trials this breakdown is by the first stimulus of the trial, as will be explained below). The data are pooled over subjects and sessions. The data analyses for positive responses will be presented first for Experiment 1 and then for Experiment 2, followed by the analyses for negative responses.
Experiment 1-positive RTs. The positiveresponse data were subjected to an analysis of variance with factors of category structure, trial type (identity match; category match), ISI, and type of instructions (pure; mixed). There was a significant main effect of trial type, $F(1,7)=12.2, p<.05$, and a Trial Type by ISI interaction, $F(1,7)=24.4$, $p<$ .01 . These effects reflect the typical advantage of identity over category matches which decreases with ISI. There was also a significant effect of instructions, with faster RT under pure instructions than under mixed, $F(1,7)=6.6, p<.05$. The three-way interaction among ISI, instruction, and category structure approached significance, $F(1,7)=5.5$, $.05<p<.06$. This marginal effect reflects the fact that under pure instructions, as compared to mixed, there was relatively little advantage for $\mathrm{S}$ categories, particularly at the shortest ISI. However, the main effect of category structure was still significant, $F(1,7)=8.6, p<.05$.

Given the marginal three-way interaction, and because the principal interest was in the effect with mixed instructions, the category-structure effect was assessed separately for mixed instructions by an analysis of variance on each trial type, with factors of ISI and category structure. For identity trials, there was a significant effect of category structure, $F(1,7)=20.7, p<.01$, with S-category RT less than $D$. Neither the effect of ISI nor the interaction was significant. [The effect of ISI was marginal, $F(1,7)$ $=3.6, \mathrm{p}<.10$.$] For category trials, there were no$ significant main effects, but there was a significant

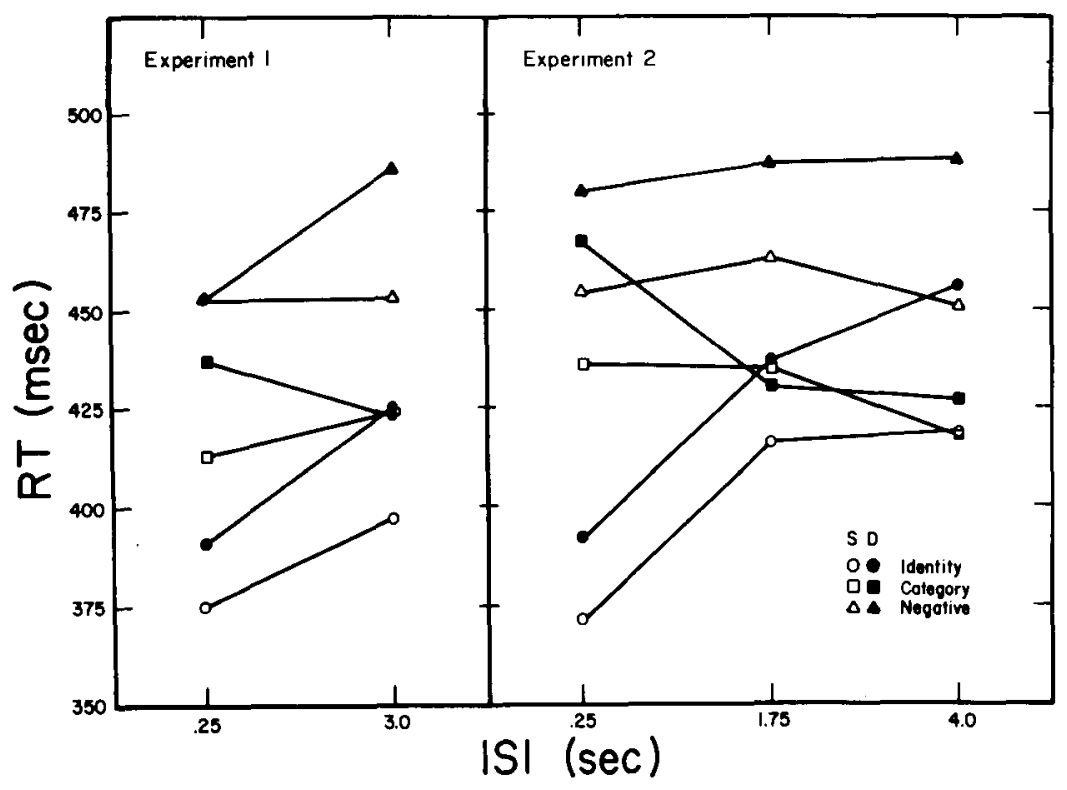

Figure 1. Mean RT for each ISI, trial type (identity match, category match, negative trial), and category structure (S or D), for mixed instructions in Experiments 1 and 2. 
Table 1

Mean Reaction Time with Pure Instructions for Each Interstimulus Interval, Instruction, and Category Structure

\begin{tabular}{|c|c|c|c|c|c|c|c|c|c|}
\hline & \multirow{3}{*}{$\begin{array}{c}\text { ISI } \\
(\mathrm{sec})\end{array}$} & \multicolumn{4}{|c|}{ Positive Responses } & \multicolumn{4}{|c|}{ Negative Responses } \\
\hline & & \multicolumn{2}{|c|}{$\begin{array}{c}\text { Category } \\
\text { Instructions }\end{array}$} & \multicolumn{2}{|c|}{$\begin{array}{c}\text { Identity } \\
\text { Instructions }\end{array}$} & \multicolumn{2}{|c|}{$\begin{array}{c}\text { Category } \\
\text { Instructions }\end{array}$} & \multicolumn{2}{|c|}{$\begin{array}{c}\text { Identity } \\
\text { Instructions }\end{array}$} \\
\hline & & $S$ & D & $S$ & D & $\mathrm{S}$ & $\mathrm{D}$ & $S$ & $\mathrm{D}$ \\
\hline Experiment 1 & $\begin{array}{l}.25 \\
3.0\end{array}$ & $\begin{array}{l}393 \\
388\end{array}$ & $\begin{array}{l}389 \\
402\end{array}$ & $\begin{array}{l}353 \\
383\end{array}$ & $\begin{array}{l}360 \\
396\end{array}$ & $\begin{array}{l}430 \\
479\end{array}$ & $\begin{array}{l}445 \\
485\end{array}$ & $\begin{array}{l}431 \\
447\end{array}$ & $\begin{array}{l}456 \\
458\end{array}$ \\
\hline Experiment 2 & $\begin{array}{l}.25 \\
1.75 \\
4.0\end{array}$ & $\begin{array}{l}411 \\
418 \\
417\end{array}$ & $\begin{array}{l}414 \\
407 \\
390\end{array}$ & $\begin{array}{l}347 \\
388 \\
418\end{array}$ & $\begin{array}{l}349 \\
371 \\
387\end{array}$ & $\begin{array}{l}440 \\
446 \\
451\end{array}$ & $\begin{array}{l}462 \\
446 \\
452\end{array}$ & $\begin{array}{l}405 \\
410 \\
434\end{array}$ & $\begin{array}{l}415 \\
423 \\
438\end{array}$ \\
\hline
\end{tabular}

ISI by Category Structure interaction, $F(1,7)=35.0$, $\mathrm{p}<.01$. As can be seen in Figure 1, this occurred because the category-structure effect was significant at the $.25-\mathrm{sec}$ ISI, $\mathrm{t}(7)=2.6, \mathrm{p}<.05$, but not at the 3.0-sec ISI.

Experiment 2-positive RTs. An overall analysis on positive responses for Experiment 2 again revealed significant effects of trial type, $F(1,7)=49.6$, $\mathrm{p}<.01$, a Trial Type by ISI interaction, $F(2,14)=$ $22.8, \mathrm{p}<.01$, and an effect of instruction type, $\mathrm{F}(1,7)=8.6, \mathrm{p}<.05$. In addition, the interaction between instructions and category structure was significant, $F(1,7)=27.5, \mathrm{p}<.01$, because the predicted advantage for $S$ categories occurred under mixed instructions but not under pure instructions. [In fact, with pure instructions there was an advantage for $\mathrm{D}$ categories at the longest $(4.0-\mathrm{sec})$ ISI, $\mathrm{t}(7)=2.80$ and $5.04, \mathrm{p}<.05$ and .01 , for identity and category instructions, respectively.]

As before, the category-structure effect with mixed instructions was investigated with a separate analysis on each type of trial, with factors of category structure and ISI. For identity trials, there was again a significant effect of category structure, $F(1,7)=20.1$, $\mathrm{p}<.01$. The effect of ISI was also significant, $\mathrm{F}(2,14)=24.4, \mathrm{p}<.01$, with $\mathrm{RT}$ increasing over ISIs, and again no interaction. For category trials, the effect of ISI was significant, $F(2,14)=3.8$, $\mathrm{p}<.05$, with RT decreasing over ISIs. Neither the category-structure effect nor the interaction was significant. However, as in Experiment 1, at the shortest ISI the category-structure effect was significant for category trials, $\mathrm{t}(7)=2.29, \mathrm{p}<.05$.

Negative RTs. The data of negative trials for each of the two experiments were subjected to an analysis of variance with factors of instruction type (pure name, pure identity, and mixed), category structure, and ISI. The category-structure variable refers to the category of the first stimulus of the trial. (The model to be described predicts an effect of the first-stimulus category under mixed instructions, but no effect is predicted when the data are broken down according to the second stimulus of the trial, nor is one observed.)
For Experiment 1, the analysis revealed a significant category-structure effect (S-category RTs less than D), $F(1,7)=5.7, p<.05$. There was a marginal effect of ISI, $F(1,7)=4.3, p<.10$, and a marginal three-way interaction among category structure, ISI, and instructions, $F(2,14)=3.0, p<.10$. Given the principal interest in RT with mixed instructions and the three-way interaction, a two-way analysis of variance was conducted on the mixedinstruction data only. The interaction was the only significant effect, $F(1,7)=6.3, p<.05$. This is because, in contrast to the category matches, the difference between negative RTs for S and D categories is significant only at the longer $(3.0-\mathrm{sec})$ ISI, $\mathrm{t}(7)=$ $2.50, \mathrm{p}<.05$.

In Experiment 2, the analysis of negative RTs revealed significant main effects of instructions (pureinstruction RTs less than mixed), $F(2,14)=8.5$, $\mathrm{p}<.01$; and category structure (S-category RTs less than D), $F(1,7)=32.6, p<.001$. There were also an Instruction by Category-Structure interaction, $F(2,14)$ $=8.0, \mathrm{p}<.01$, and an Instruction by ISI interaction, $F(4,28)=3.7, p<.05$. The former interaction reflects the fact that category-structure effects were robust under mixed instructions but not pure. The latter indicates an increase in RT over ISIs under pure identity instructions only. The analysis on the mixed-instruction $\mathrm{RTs}$ revealed only a significant category-structure effect, $F(1,7)=45.8, p<.01$, and no other main effects or interactions.

The foregoing analyses were conducted on data pooled over all sessions in order to provide a larger data base. Examination of the data by individual sessions is also of interest, however, for consideration of practice effects. Such an examination reveals the general decrease in RT with practice (i.e., over sessions) usually observed in such experiments. In addition, it is noteworthy that the magnitude of the category-structure effect tends to increase over successive mixed-instruction sessions, particularly for the category matches (at ISIs where the effect occurs) and with a similar, but small, trend for negative trials. This trend is consistent with the idea that the category-structure effect reflects the subject's 
long-term knowledge about the stimulus pool, since this knowledge would tend to increase with longer participation in the experiment.

\section{Discussion}

The results of Experiments 1 and 2 generally replicate the findings of Klatzky and Stoy (1974) and extend them beyond manipulations of visual similarity to the semantic domain. As noted above, the principal implication of the category-structure effect obtained in these studies is that subjects' performance on a trial of the comparison task reflects their knowledge of the category from which the experimental stimuli come. In both experiments, under mixed instructions, an effect of category structure was obtained in all three types of trials-identity matches, category matches, and negatives. This effect reflects the fact that when the stimuli in a category are more similar semantically, RT is faster. The category-structure effect is particularly noteworthy for identity matches, since all identity-match trials involve two identical stimuli. If subjects used only knowledge pertaining to the immediate trial, there should be no difference in identity-match RTs to stimuli from S and $D$ categories. Thus, the observed identity-match RTs, in particular, indicate that the subjects perform the task in the larger context of their long-term knowledge of the stimulus pool.

The evidence that subjects use long-term knowledge about the pictures forms the basis for a model of processing in this task. This model is an extension of one presented in Klatzky and Stoy (1974; also cf. Kroll, 1975). Its primary assumption is that on each trial of the task, after the first picture is presented, the subject accesses information about its category in long-term memory and generates, or activates, a representation of the pictures that might appear as second stimuli, if the trial is positive. The subject is also assumed to maintain a short-term trace of the first stimulus which is lost over time. When the second stimulus appears, the subject compares it to the codes he has in memory; the outcome of these comparisons determines the response.

The model further assumes that the subject's capacity to generate codes from long-term memory is limited; it is this capacity limitation which produces the category-structure effect. More specifically, with mixed instructions, the subject must generate two codes-one representing each of the stimuli that might appear. When these codes represent similar stimuli (i.e., when the stimuli are members of an $\mathrm{S}$ category), capacity is more efficiently allocated (as will be explained below), leading to shorter RTs than when the stimuli represented are dissimilar (from a D category).

Category-structure effects should occur only under mixed instructions, according to the model, because only then does the subject generate codes represent- ing two stimuli. With pure instructions, the subject needs to anticipate just one stimulus, since the first stimulus on a trial predicts a single second stimulus if the trial is to be positive (one identical to the first with pure identity instructions and the alternate member of the category with pure category instructions). There is therefore no reason to propose that the subject will generate both category members, and hence no prediction of a category-structure effect.

Given the assumption that codes generated from long-term memory are used in the comparison task, a question arises as to the nature of these codes. The assumption made here is that the long-term code of a stimulus consists of semantic features, like those suggested in certain models of semantic memory (Smith, Shoben, \& Rips, 1974). Such features might describe visual characteristics of the stimulus (e.g., "has feathers," for a robin). However, they need not be so restricted and might also pertain to nonphysical qualities (e.g., "can fly"). The nondifferentiation between semantic features pertaining to physical and nonphysical properties is suggested by the present effects when category structure is manipulated on a semantic rather than visual basis. The work of Rosch et al. (1976) is also relevant here, in which subjects were asked to produce attributes of common categories from long-term memory. The data suggest that it is difficult to discriminate between category-structure manipulations based on physical, nonphysical, or motoric properties, for the attributes covary.

The assumption that the long-term codes are made up of features receives support from certain studies indicating feature-based codes in the stimuluscomparison task. The above-cited experiment of Klatzky and Stoy (1974), in which subjects responded to mirror images as rapidly as to identical pictures, indicates that the codes used for identity matches are made up of features. Additional data suggesting the representation of stimuli by features comes from work of Kroll, Kellicutt, Berrian, and Kreisler (1974). They conducted a letter-comparison task with temporally separated letter pairs that might or might not differ in color on each trial. One group of subjects had to perform a same/different judgment of the letters on the basis of names; a second group not only performed the judgment, but also recalled the color of the first letters on each trial. Subjects in the latter group were slower when the letter pairs on a given trial differed in color than when their color was constant. In contrast, RT of the subjects who did not recall color was unaffected by color differences, after the briefest ISI. These results suggest that subjects for whom color information was irrelevant could discard that information over time, retaining a more abstráct stimulus representation that conveyed only features relevant to their decision.

An alternative possibility that might be raised is 
that the generated codes are nominal. That is, the hypothesis might be raised that agreement/disagreement between names of category inembers constitutes the operative difference between $S$ and $D$ categories in these experiments. However, several facts argue against such a proposal. First, the previously described work of Parks and Kroll (1975) supports the idea that subjects generate nonnominal codes in this task, even with stimuli that are relatively impoverished semantically (letters). Second, the category-structure variable in Experiment 2 did not explicitly manipulate stimulus names; yet, category-structure effects were obtained. Moreover, in both experiments, experimenter and subject repeatedly named pictures in both types of categories with the same name, which should have provided subjects with common names even for D-category members. Although these facts do not make it possible to absolutely rule out the nominal hypothesis, they render it improbable.

The short-term code also proposed by the model to be used in this task, that is, the trace of the first stimulus presented on a trial, is similarly assumed to be composed of features. (Evidence for this assumption has been discussed above.) The model does not dictate the nature of this trace more specifically. It could consist of features that are qualitatively different from those in the long-term code (e.g., concrete visual features). Alternatively, the short-term trace could simply have more features than the longterm code. The model's principal distinction between these codes, in any case, is that the time required for a comparison between the second stimulus of a trial and a memory code is less for the short-term code than for the long-term.

The model of processing in this task will now be presented in more detail. It will also be described quantitatively.

\section{Model of the Comparison Task}

The model describes processing on a single trial of the comparison task, under mixed instructions. During this trial, the subject is first presented with a pictorial stimulus, denoted $\mathrm{P}_{\mathrm{i}}$. The presentation interval is followed by an ISI, then by presentation of a second picture-to be called the test picture. The test picture may be $P_{i}$, in which case the trial is an identity match; the alternate member of the $\mathrm{P}_{\mathrm{i}}$ category, denoted $\mathrm{P}_{c}$, for a category match; or some other picture, $P_{n}$, for a negative trial.

Assumptions about coding. It is assumed that three codes are used to represent stimuli in memory: Immediately after presentation of $\mathrm{P}_{\mathrm{i}}$, a short-term trace is formed; this loses information over time. In addition, the subject generates two codes from long-term memory, to represent $\mathrm{P}_{i}$ and $\mathrm{P}_{c}$; this occurs immediately after $\mathrm{P}_{i}$ presentation.

Assumptions about the comparison process. When the test picture appears, the subject compares it in parallel to all the codes he has in memory, including those generated from long-term memory and the short-term trace of $\mathrm{P}_{i}$, if available. This comparison process has four possible outcomes: (1) If the trial is an identity match, the comparison between the test picture and the short-term code of $\mathrm{P}_{i}$ may yield the response. This is assumed to be a relatively fast process (cf. Posner, 1969); however, its probability decreases as the interval following first-stimulus presentation increases, due to loss of features from the short-term trace. (2) If the trial is an identity match and outcome 1 does not occur, the test picture will be matched with the long-term code of $P_{j}$. This is a slower process than (1). (3) If the trial is a category match, the test picture and long-term code of $\mathrm{P}_{c}$ will be matched. (4) If no match is made between the test picture and any available code (listed in 1-3), a negative response is output.

The comparison process involving long-term codes will now be described in more detail: First, it is assumed that the long-term code is composed of a set of weighted features. Each feature has either a low or a high weight, depending on its value to the comparison process. Second, each comparison between the test picture and a long-term code is assumed to begin with a counter set at some value (representing response bias). The counter has an upper value, which if passed elicits a positive response, and a lower value, which if passed elicits a negative decision. The subject cycles through a series of feature comparisons, on each of which the following occurs: (1) A feature is sampled from the available set in memory. (2) The test picture is examined for the presence of the selected feature. (3) The counter is adjusted upward by the weight of the selected feature if that feature is matched with the picture, or adjusted downward if it is not matched. This process continues until either the upper or lower value of the counter is passed. If the upper value is passed, a positive response is elicited immediately. If the lower value is passed, the processor waits for the outcome of all other comparisons. When all comparisons are negative, a negative response is output. (This model is similar to one proposed by Buckley and Gillman, 1974, for judgments of numerosity.)

The process of comparing the test picture to a generated (long-term) code can be specified in more detail as follows. Let the number of features in the generated code representing a single picture be $F$. Some of these features have a high weight; however, it is assumed that only a limited number of features (call the number $w$ ) can be given high weights at any one time. (This represents the limit on processing capacity.) The remaining $F-w$ features receive low weights. Since the processing of a highly weighted feature has a greater effect on the counter than that of a low-weight feature, the number of features that 
must be processed, and thus the expected time to complete the comparison process, decreases as the number of highly weighted features in the code increases. (See Appendix 1.)

When two codes are generated from long-term memory (as is necessary to represent both $P_{i}$ and $P_{c}$ ), two $F$ features will be activated. Since these codes represent pictures from the same category, some number of features, $\mathrm{k}$, will be common to both codes. Given the capacity limitation, it will be to the subject's advantage to assign high weights to the $\mathrm{k}$ common features, since these will be used in more than one comparison. (This represents the allocation of capacity efficiently, as discussed above.) The remaining high weights can be distributed to features that represent just one picture. It will generally be to the subject's advantage to distribute these additional weights equally over the two generated codes, since both pictures are equally possible as the test item. In this case, of the $\mathrm{F}$ features representing each picture, $k+(w-k) / 2=(k+w) / 2$ will have high weights. This value increases with the number of common features, $\mathrm{k}$, and, as shown in Appendix 1, the greater this value (i.e., the more features representing a picture that have high weights), the faster the comparison process will proceed.

In terms of the present experiments, since the pictures in an S category are more similar, they should have more common features than those in a D category, and the value of $\mathrm{k}$ should be higher. Thus, the comparison time for long-term codes representing $\mathrm{S}$-category stimuli should be less. Let the comparison time be $\mathrm{L}_{\mathrm{s}}$ and $\mathrm{L}_{\mathrm{d}}$ for comparisons of long-term codes representing $\mathrm{S}$ - and $\mathrm{D}$-category stimuli, respectively, with $\mathrm{L}_{\mathrm{s}}<\mathrm{L}_{\mathrm{d}}$.

The foregoing describes the situation in which the subject assigns weights to the two codes equitably. However, he could choose to emphasize one code at the expense of the other. Post hoc evaluation of the data suggests that this occurs in one case (with $\mathrm{D}$ categories, at long ISIs), where the subject appears to expend additional processing capacity to anticipate $P_{c}$. This is accomodated within the model by assuming that when the first stimulus of the trial is from a D category, and the subject has a long ISI, he is motivated to highly weight more features. Moreover, he assigns the added weights to features exclusive to $P_{c}$. This will lead to. a particularly fast comparison with the long-term code representing $P_{c}$ in this case; this fast time is denoted $L_{d, f}$, where $\mathrm{L}_{\mathrm{d}, \mathrm{f}}<\mathrm{L}_{\mathrm{d}}$.

The comparison process between the test picture and short-term trace will now be described: This trace follows $P_{i}$ presentation, and features are lost from it with time. The comparison at time $t$ after $P_{i}$ presentation ( $t$ is determined by the ISI) succeeds with probability $\pi(t)$, and if successful, the compar- ison takes time I, less than the time for comparison with a long-term code. The probability of a successful short-term comparison, $\pi(t)$, is a decreasing function of t. (See Appendix 2.)

The general model. The model assumes that identity matches can be based on a comparison between the test picture and short-term trace of $\mathrm{P}_{\mathrm{i}}$ [with probability $\pi(t)$ ] or the long-term code of $P_{i}$. The former has temporal parameter $\mathrm{I}$; the latter, $\mathrm{L}_{\mathrm{s}}$ or $\mathrm{L}_{\mathrm{d}}$, depending on the stimulus category. Thus, the expected $\mathrm{RT}$ for identity trials at time $\mathrm{t}$ after presentation of $P_{i}$ is:

$$
R T(t)=\pi(t) I+[1-\pi(t)] L_{j}+m .
$$

Here $L_{j}$ can be $L_{s}$ or $L_{d}$, depending on the stimulus category, and $m$ represents the combined times for encoding the test picture and responding. At long ISIs, we can assume $\pi$ is zero. At an ISI of zero, $\pi$ should be 1.0 .

Category matches, according to the model, are based on the long-term code of $P_{c}$. Thus, the expected RT is:

$$
\mathrm{RT}=\mathrm{L}_{\mathrm{j}}+\mathrm{m} \text {. }
$$

Here, $L_{j}$ is the time for the comparison with the longterm code. Again, $\mathrm{j}$ represents $\mathrm{s}$ if the stimuli are from an $\mathrm{S}$ category. However, if they are from a $D$ category, two parameters are necessary because of the special case noted above. Thus, for short ISIs, $L_{j}$ is $L_{d}$, but at longer ISIs, it is $L_{d, f}$, where $L_{d, f}$ $<\mathrm{L}_{\mathrm{d}}$, as described previously.

In the case of negative responses, the processor must wait for the output of the slower of the two long-term comparisons (using the codes for $P_{i}$ and $P_{c}$ ) before responding. Thus, the expected $R T$ is:

$$
R T=\operatorname{maximum} L_{j}+m^{\prime} .
$$

Here, $m^{\prime}$ is the negative-response equivalent of $m$. Generally, negative responses are slower in the same/ different tasks (Nickerson, 1972); thus, we can assume $m^{\prime}>m$. The maximum $L_{j}$ will be $L_{s}$ if the first stimulus of the trial is from an $S$ category. It will be $L_{d}$ if the first stimulus is from a $D$ category, regardless of the ISI (since $L_{d}>L_{d, f}$ ). Note that the predicted negative RT depends only on the firststimulus category, not that of the second, test picture. (It has previously been indicated that actual RTs similarly do not depend on the second-picture category.)

Several ordinal relationships are known about the parameters of Equations 1-3, specifically: $\mathrm{I}<\mathrm{L}_{\mathrm{s}}<$ $\mathrm{L}_{\mathrm{d}} ; \mathrm{L}_{\mathrm{d}, \mathrm{f}}<\mathrm{L}_{\mathrm{d}} ; \mathrm{m}<\mathrm{m}^{\prime}$. For this reason, the parameters can be recoded as follows: $\mathrm{L}_{\mathrm{s}}=\mathrm{I}+\alpha$; $\mathrm{L}_{\mathrm{d}}$ $=\mathrm{L}_{\mathrm{s}}+\beta=\mathrm{I}+\alpha+\beta ; \mathrm{L}_{\mathrm{d}, \mathrm{f}}=\mathrm{L}_{\mathrm{d}}-\delta=\mathrm{I}+\alpha$ 
Table 2

Predictive Equations

\begin{tabular}{|c|c|c|c|c|c|}
\hline \multirow[b]{2}{*}{$\begin{array}{l}\text { Trial } \\
\text { Type }\end{array}$} & \multirow[b]{2}{*}{$\begin{array}{l}\text { Category } \\
\text { Structure }\end{array}$} & \multicolumn{4}{|c|}{ ISI } \\
\hline & & $\begin{array}{c}\text { Zero } \\
(\pi=1)\end{array}$ & $\begin{array}{c}\text { Short } \\
(.25 \mathrm{sec})\end{array}$ & $\begin{array}{l}\text { Moderate } \\
(1.75 \mathrm{sec})\end{array}$ & $\begin{array}{c}\text { Long } \\
(4.0 \sec , \pi=0)\end{array}$ \\
\hline Identity & $\begin{array}{l}\mathrm{S} \\
\mathrm{D}\end{array}$ & $\begin{array}{l}I+m \\
I+m\end{array}$ & $\begin{array}{l}\pi \mathrm{I}+(1-\pi)(\mathrm{I}+\alpha)+\mathrm{m} \\
\pi \mathrm{I}+(1-\pi)(\mathrm{I}+\alpha+\beta)+\mathrm{m}\end{array}$ & $\begin{array}{l}\pi I+(1-\pi)(I+\alpha)+m \\
\pi I+(1-\pi)(I+\alpha+\beta)+m\end{array}$ & $\begin{array}{l}\mathrm{I}+\alpha+\mathrm{In} \\
\mathrm{I}+\alpha+\beta+\mathrm{m}\end{array}$ \\
\hline Category & $\begin{array}{l}\mathrm{S} \\
\mathrm{D}\end{array}$ & $\begin{array}{l}\mathrm{I}+\alpha+\mathrm{m} \\
\mathrm{I}+\alpha+\beta+\mathrm{m}\end{array}$ & $\begin{array}{l}\mathrm{I}+\alpha+\mathrm{m} \\
\mathrm{I}+\alpha+\beta+\mathrm{m}\end{array}$ & $\begin{array}{l}\mathrm{I}+\alpha+\mathrm{m} \\
\mathrm{I}+\alpha+\beta-\delta+\mathrm{m}\end{array}$ & $\begin{array}{l}\mathrm{I}+\alpha+\mathrm{m} \\
\mathrm{I}+\alpha+\beta-\delta+\mathrm{m}\end{array}$ \\
\hline Negative & $\begin{array}{l}\mathrm{S} \\
\mathrm{D}\end{array}$ & $\begin{array}{l}I+\alpha+m+\epsilon \\
I+\alpha+\beta+m+\epsilon\end{array}$ & $\begin{array}{l}1+\alpha+m+\epsilon \\
1+\alpha+\beta+m+\epsilon\end{array}$ & $\begin{array}{l}\mathrm{I}+\alpha+\mathrm{m}+\epsilon \\
\mathrm{I}+\alpha+\beta+\mathrm{m}+\epsilon\end{array}$ & $\begin{array}{l}I+\alpha+m+\epsilon \\
I+\alpha+\beta+m+\epsilon\end{array}$ \\
\hline
\end{tabular}

Table 3

Parameter Estimates

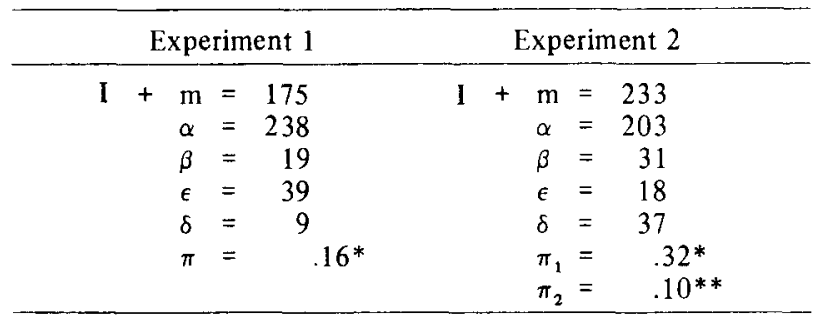

${ }^{*} I S I=.25 \mathrm{sec}$.

$* * I S I=1.75 \mathrm{sec}$.

$+\beta-\delta ; m^{\prime}=m+\varepsilon$. The equations in terms of the recoded parameters, used to predict $R T$ for various experimental cases are shown in Table 2 .
Parameter estimates were made from these equations using the method of partial data (Restle \& Greeno, 1970). The parameters are shown for Experiments 1 and 2 in Table 3, and the predicted RTs are shown for Experiment 2 in Figure 2. (For these predictions, $r^{2}=.94$.) Since fewer predictions were possible for Experiment 1 due to the use of one fewer ISI value, the predictions for that study are not shown. In general, however, the model provided as good an account of Experiment 1 as of Experiment 2 (except for predicting a discrepant high value for negative-response RT to D-category stimuli at the shortest ISI). Thus, the model provides predictions that are in general accord with the data of the first two studies. Moreover, the parameter estimates for the two studies are similar, and in Experiment 2 the

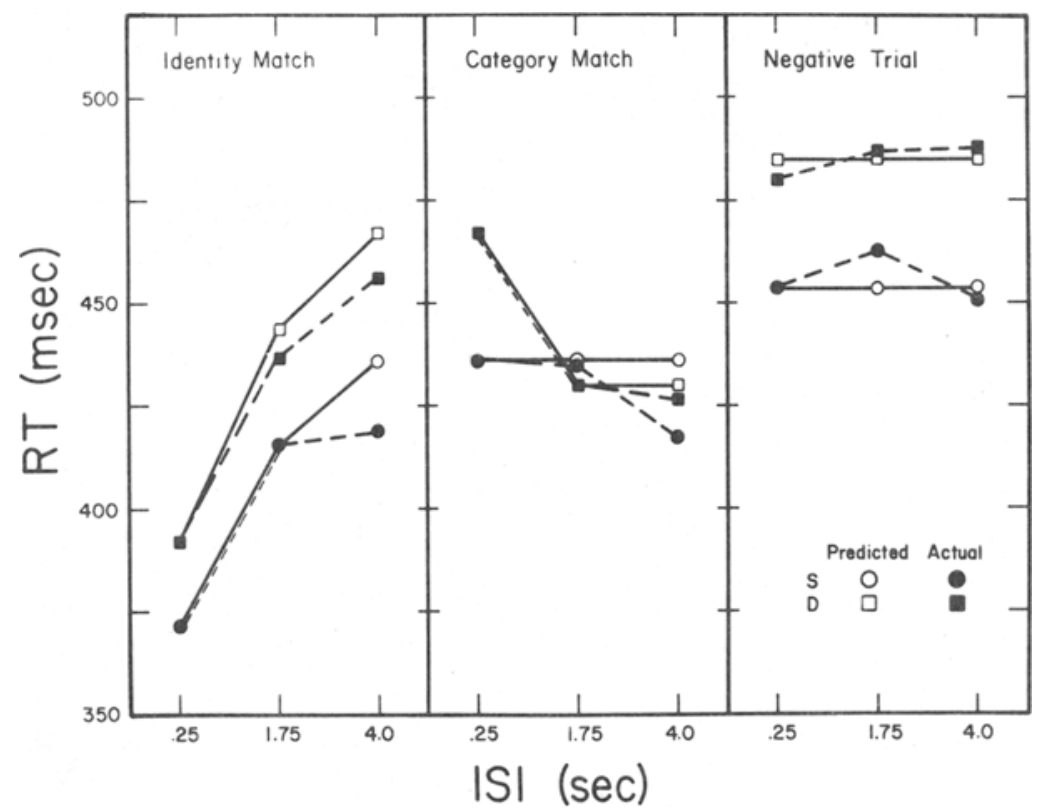

Figure 2. Observed (closed points) and predicted (open points) RTs with mixed instructions in Experiment 2, for each ISI, trial type, and category structure. Where there is a single closed point, the condition was used in parameter estimation and the RT is therefore predicted perfectly. 
two independent estimates of the value of $\pi$ (one for each ISI) decrease as expected with increasing ISI.

Note that this model can also account for past data using this same/different task by adopting a single parameter for the time for long-term comparisons. Similarly, it can account for the absence of category-structure effects under pure instructions, since the expectation of a single test stimulus eliminates the need for weighting common features and thus eliminates the source of the effect. Finally, an account of the finding in Experiment 2, of an advantage for D-category stimuli under pure instructions, can also be offered. In this case, the subject forms expectations for just one category member and may, given a long ISI, assign additional high weights to features that maximally differentiate the category members. There are more such features for Dcategory stimuli, and thus their long-term codes will lead to faster comparisons.

As is apparent from the foregoing discussion, this model hypothesizes the use of subject-controlled strategies for allocating processing capacity, in order to account for such results as changes in the categorystructure effect over ISIs (for category matches) and reversal of the effect under pure instructions in Experiment 2. However, it is presently inadequate to predict precisely the conditions under which a given strategy will be used.

\section{EXPERIMENT 3}

The model just described predicts an effect of category structure on identity-match RT only when the short-term trace of the first picture presented does not always yield a positive response. However, if the short-term trace is consistently adequate for responding, there should be no category-structure effect on identity RT. This prediction could not be tested in the first two experiments, because the smallest ISI used $(250 \mathrm{msec})$ undoubtedly allows some changes in the short-term code to occur (as evidenced by the values of $\pi$, which are not close to 1.0). For this reason, a third experiment was run, in which the ISI was zero. Under these conditions, a match between the test picture and short-term trace should consistently occur (i.e., $\pi=1.0$ ), and there should be no difference between identity-match RT for S- and D-category stimuli. However, category structure should still affect category-match and negative RTs, for these will still depend on codes generated from long-term memory.

\section{Method}

Subjects. The subjects were 10 students at the University of California, Santa Barbara.

Stimuli. The stimuli and counterbalancing of their assignment to subjects were the same as in Experiment 1.
Procedure. Each subject took part in a single session of three practice trials and 120 experimental trials. Only mixed instructions were used. After hearing the instructions, the subject was shown the experimental pictures and allowed to study them until he felt he could reproduce them. Then he was asked to draw both mem. bers of each category so that they could be distinguished from one another. When he could do so, the trials began.

The procedure for a single trial was the same as that of Experiment 1 , with the following exceptions: The first picture appeared to the left of fixation; its duration was $250 \mathrm{msec}$. The second picture followed immediately after the first, to the right of fixation. This lateral presentation was used to minimize masking.

\section{Results and Discussion}

As before, the data are mean RTs over subjects, excluding error and practice trials. These are shown in Table 4. Again, error rates were low, ranging from $1.6 \%$ to $6.7 \%$ with a mean of $3.0 \%$. The positive data were submitted to an analysis of variance, with factors of category structure and trial type. There were significant effects of trial type, $F(1,9)=42.6$, $\mathrm{p}<.001$, and category structure, $\mathrm{F}(1,9)=5.6, \mathrm{p}<$ .05 , and a significant interaction, $F(1,9)=7.2$, $\mathrm{p}<.05$. The interaction reflects the fact that category structure was significant for category-match trials, $\mathrm{t}(9)=2.71, \mathrm{p}<.05$, but not for identitymatch trials, as predicted. The difference in RTs for negative trials with first stimulus from an $S$ category vs. a D category was not significant.

The data of this experiment show the predicted pattern of results for identity and category trials, with category-structure effects for the latter but not the former. Although the same effect was not significant for negative trials, it was in the predicted direction. The lack of effect may represent the use of a strategy for negative responses not assumed by the model.

\section{GENERAL DISCUSSION}

The present series of experiments demonstrates that same/different judgments about temporally separated pictures of objects utilize information about the object category, even when such information is not essential for the judgment. This general finding has been incorporated into a model which proposes that the subject accesses categorical information and generates stimulus representations in anticipation of the pictures that might appear on the trial. Limited capacity for generating these represen-

Table 4

Mean Reaction Time in Experiment 3 for

Each Category Structure and Trial Type

\begin{tabular}{lccc}
\hline & $\begin{array}{c}\text { Identity } \\
\text { Match }\end{array}$ & $\begin{array}{c}\text { Category } \\
\text { Match }\end{array}$ & $\begin{array}{c}\text { Negative } \\
\text { Trial }\end{array}$ \\
\hline S Categories & 435 & 494 & 577 \\
D Categories & 448 & 549 & 594 \\
\hline
\end{tabular}


tations gives rise to effects of stimulus-category structure on RT. The model was developed quantitatively and used to predict RTs that were in general accord with the observed RT data in two experiments. Its predictions were less adequate but at least partially supported by a third experiment.

The suggestion that same/different judgments with temporally separated stimuli utilize codes generated from long-term memory has been made by other researchers as well. The work of Kroll and his colleagues has been reviewed above; the present results are in agreement with Kroll's account of the same/ different judgments.

In a similar area of investigation, Rosch (1975) has also obtained evidence that information in longterm memory is accessed and used in same/different judgments. She used a variant of Posner's task in which the two stimuli to be compared are presented simultaneously, but preceded by a verbal prime that names their category. Rosch has found that the prime facilitates comparisons of typical instances of the category and inhibits comparisons of atypical instances, suggesting that subjects use the prime to access the category and activate its typical members in advance of stimulus presentation. Further, as in the present studies, Rosch has found that priming is effective under instructions like the present mixed instructions but not like the pure instructions.

In suggesting that there are different levels of representation that may be used for comparing pictorial stimuli, the present model is also in agreement with work of Bartram $(1974,1976)$. He has proposed that stimuli like the present ones may be compared on two or more levels, including a pictorial level and a more abstract semantic level, similar to the present short-term and long-term codes, respectively.

In summary, a growing body of research suggests that subjects are able to make comparisons of visual stimuli on the basis of several types of information, including semantic information from long-term memory. In particular, the present research indicates that a simple distinction between visual and verbal coding does not provide a complete account of performance in the task developed by Posner (1969), at least when the stimuli are pictures. The present research further indicates that the nature of the codes used for picture processing is under subjective control, for evidence was found in these studies that subjects adjust their picture codes according to the experimental conditions.

\section{APPENDIX 1}

Suppose that a long-term memory code has $\mathrm{M}$ features, $\mathrm{n}$ of which have a high weight. We wish to show that as $\mathrm{n}$ increases, the expected time to complete the comparison process decreases. Let the proportion of high-weight features, $n / M$, be called $\mathrm{p}$. These features have weight $\mathrm{x}$; the remaining features have a lower weight $y$.
Define a variable $F$ as the number of features with a high weight that are selected in $g$ samples. The variable $F$ is binominai $(g, p)$ distributed (assuming features are sampled with replacement). Define $\mathrm{D}_{\mathrm{g}}$ as the total of the increments (or decrements) in the counter after $g$ samples. Then $D_{g}=$ $F x+(g-F) y$. And the expected value of $D_{g}, E\left(D_{g}\right)=$ $x E(F)+y E(g-F)=x(p g)+y g-y p g=g[p(x-y)$ $+y]$.

Let the distance between the starting value of the counter and the criterion value for the response be $C$. When the expected value of $D_{g}=C$, then $C=g^{\prime}[p(x-y)+y]$ for some number of samples, $g^{\prime}$. And $g^{\prime}=C /[p(x-y)$ $+y]$. Since $(x-y)$ is greater than zero, $g^{\prime}$ will be a monotonic decreasing function of $\mathrm{p}$. A similar demonstration holds for any value $C^{\prime}$ that might be the first value of the counter to exceed $C$.

\section{APPENDIX 2}

The model assumes that features are lost from the shortterm trace of the first stimulus presented, over time. This results in a decrease in the probability of a successful comparison between the second picture and short-term trace, over time. The comparison is assumed to take time 1 . One way to model these assumptions is to begin by assuming that the short-term code of the first stimulus is initially composed of some number of features, G. During each unit of time, a constant proportion $(1-x)$ of these features are discarded. Then, at time $t$ after first-stimulus offset, the remaining features number $\mathrm{Gx}^{1}$. The comparison process between the second picture and short-term code is assumed to involve parallel comparisons of their features; this takes time I. Given that the trial is an identity match (i.e., a successful comparison is possible), the comparison yields a positive output if the number of matching features (which will be the number of features in the short-term code) exceeds some criterion value, $k$. The value $k$ is determined by a random variable on each trial. If the variable is uniformly distributed over an interval from some minimum value a, to $G$, then $\pi(t)$ is the probability that $\mathrm{k} \leqslant \mathrm{Gx}^{\mathrm{l}}$, which is:

$$
\pi(t)=\begin{aligned}
& \left(G x^{t}-a\right) /(G-a) \text { if } G x^{t}>a \\
& 0 \text { otherwise }
\end{aligned}
$$

This monotonically decreases as $t$ increases.

\section{REFERENCES}

Bartram, D. J. The role of visual and semantic codes in object naming. Cognitive Psychology, 1974, 6, 325-356.

BARTRAM. D. J. Levels of coding in picture-picture comparison tasks. Memory \& Cognition. 1976, 4, 593-602.

Buckley, P. B., \& Gillman, C. B. Comparisons of digit and dot patterns. Journal of Experimental Psychology, 1974, 103, 1131.1136.

Cooper, L. A., \& Shepard, R. N. The time required to prepare for a rotated stimulus. Memory \& Cognition, 1973, 1, 246-250.

Klatzky, R. L., \& Stoy, A. M. Using visual codes for comparisons of pictures. Memory \& Cognition, 1974, 2, 727-736.

Kroll, N. E. A. Visual short-term memory. In D. Deutsch \& J. A. Deutsch (Eds.), Short-term memory. New York: Academic Press, 1975.

Kroli. N. E. A.. Kellicutt, M. H. Berrian, R. W., \& KREISLER. A. F. Effects of irrelevant rolor changes on speed 
of visual recognition following short retention intervals. Journal of Experimental Psychology, 1974, 103, 97-106.

LyoNs, J. Introduction to theoretical linguistics. London: Cambridge University Press, 1968.

NICKERSON, R. S. Binary-classification reaction time: A review of some studies of human information-processing capabilities. Psychonomic Monograph Supplements, 1972, 4(Whole No. 65) $275-318$.

Parks, T. E., \& KRoll, N. E. A. Enduring visual memory despite forced verbal rehearsal. Joumal of Experimental Psychology: Human Learning and Memory, 1975, 1, 648-654.

Posner, M. I. Abstraction and the process of recognition. In J. T. Spence \& G. H. Bower (Eds.), Advances in learning and motivation (Vol. 3). New York: Academic Press, 1969.

Posner, M. I., Boies, S. J., Eichelman, W. H., \& Taylor, R. L. Retention of visual and name codes of single letters. Journal of Experimental Psychology, 1969, 79 (1, Pt. 2).

Posner, M. I., \& Mitchell, R. F. Chronometric analysis of classification. Psychological Review, 1967, 74, 392-409.
Restle, F., \& GReENo, J. G. Introduction to mathematical psychology. Reading, Mass: Addison-Wesley, 1970.

Rosch, E. Cognitive representations of semantic categories. Journal of Experimental Psychology: General, 1975, 104, 192-233.

Rosch, E., Mervis, C. B., Gray, W., Johnson, D., \& Boyes-Brafm, P. Basic objects in natural categories. Cognitive Psychology, 1976, 8, 382-439.

Smith, E. E., Shoben, E. J., \& Rips, L. J. Structure and process in semantic memory: A featural model for semantic decision. Psychological Review, 1974, 81, 214-241.

TVersky, B. Pictorial and verbal encoding in a short-term memory task. Perception \& Psychophysics, 1969, 6, 225-233.

(Received for publication June 27, 1977; revision accepted November $30,1977$. ) 